\title{
PROPUESTA METODOLÓGICA PARA ANALIZAR LA SITUACIÓN ECONÓMICA DE POBLACIONES ARTESANALES
}

\author{
Sandra Nadyedja Landázuri Espinoza ${ }^{1 *}$; Norma Janet Montenegro Cazares ${ }^{1}$ \\ ${ }_{1}^{1}$ Pontificia Universidad Católica del Ecuador, Sede Ibarra, Escuela de Negocios y Comercio Internacional \\ *Autor para correspondencia: e-mail: snlandazuri@pucesi.edu.ec
}

Recibido: 2017/10/31

Aprobado: 2018/06/07

DOI: https://doi.org/10.26621/XIV18.2018.06.A02.PUCESI.2550.6684

\section{RESUMEN}

Las poblaciones artesanales cuentan con características propias y diversas, una de ellas es que son unidades familiares y no cuentan con la planificación administrativa, contable y publicitaria, convirtiéndose esto en una deficiencia para el desarrollo de las mismas. Esta investigación tiene como propósito diseñar un modelo metodológico, para el análisis económico de poblaciones artesanales, el modelo está constituido por cuatro fases que, a la vez, contienen acciones en las que se integran datos cualitativos y cuantitativos que se toman de la población de estudio, formando una red de relación entre ellas que dan como resultado un modelo que, en su fase final, permite describir estrategias que ayuden a impulsar el desarrollo económico de la población.

Palabras Claves: Metodología, artesanos, recursos, procesos, producto.

\begin{abstract}
Artisanal populations possess their own and diverse characteristics, one of said characteristics is the fact that they are family units and do not have administrative, accounting and advertising planning, something that is becoming a deficiency for their development. This research has the purpose of designing a methodological model, for the economic analysis of artisanal populations. The model consists of four phases, which at the same time, contain actions where qualitative and quantitative data are integrated, taken from the study population, forming a network of relationships between them. The result will be a model that, in its final phase, will be able to describe strategies that help boost the economic development of the population.
\end{abstract}

Keywords: Artisans, resources, processes, product 


\section{INTRODUCCIÓN}

En los países de América Latina es común encontrar poblaciones artesanales que se dedican a diversas actividades como: bordados, tallados en madera, tagua, tejidos en fibra, cuero, paja toquilla, cerámicas entre otras. Al reconocer la importancia de la producción artesanal, la UNESCO (2010) manifiesta.

Que es una forma importante de expresión cultural porque refleja la estética, el simbolismo y la visión del mundo de las comunidades productoras. Como forma comercial de la diversidad cultural, la artesanía se ha convertido en una fuente importante de ingresos y empleos en muchas partes del mundo (p.180).

La artesanía se entiende como la labor de creación o decoración, de manera predominantemente manual y artística, de objetos de utilidad práctica para la sociedad (Oliveras \& Albanes, 2012, p.4). Las poblaciones artesanales son producto de una tradición, de la herencia cultural de los pueblos, que se transmiten de generación en generación y, como tales, nacen en el núcleo familiar sin estudio o planificación alguna. En este sentido Leff, (2003 citado en Pacheco, 2009) expresa que: "La preservación de la identidad étnica y su autonomía cultural contribuyen a la conservación y desarrollo del potencial productivo de su ambiente". Una de las características de estas poblaciones es la atracción que sienten los turistas por visitarlas, por lo que las expectativas de crecimiento en el sector turístico van en aumento, contribuyendo a la generación de empleo directo e indirecto y aportando en la dinamización de la economía en un país.

Los negocios artesanales forman parte del tejido de las micro, pequeñas y medianas empresas (Mipymes). En éstas, la administración es empírica, no especializada e informal, afectando a su desarrollo y crecimiento. Según Derby, y Brooksbank (1997, 1996 citados por Culkin \& Smith, 2000. p.7) la diferencia está en que el administrador de la pequeña empresa no cuenta con canales especializados para la toma de decisiones complejas; por lo tanto, es el administrador/propietario quien asume total responsabilidad sobre tecnología, comunicación, banca, papelería, etc.

En el caso del Ecuador, de cada 100 establecimientos, 99 encajan en la Mipymes (INEC, 2010). Además, considerando lo declarado por la Junta Nacional de Defensa del Artesano, el sector artesanal aporta con el $1 \%$ al PIB nacional. Sin embargo, los pequeños negocios carecen de un buen manejo de costos, canales de comercialización y marketing entre otras deficiencias que se observan.

La globalización obliga a las empresas a ser altamente competitivas, en busca de establecerse en un mercado y permanecer en él, siendo la clave del éxito o del fracaso la implementación de una estrategia adecuada (Landázuri - Espinoza, Montenegro - Cazares .2018. p.2).

Al respecto Castro (2010) manifiesta que la estrategia debe ser alcanzable y realizable, respondiendo a sus recursos, capacidades y características propias de la empresa (P.248).

Frente a ésta realidad, se plantean las preguntas: ¿Necesitan las poblaciones artesanales un asesoramiento en temas administrativos y financieros para el crecimiento de sus unidades productivas? y ¿Cuál es la proyección de la producción de los artesanos, frente a las rentas obtenidas por la comercialización de sus productos?

En este contexto, es importante diagnosticar la situación económica de la población de estudio, para proponer estrategias tendientes a mejorar la realidad actual de sus habitantes

El objetivo principal de la investigación es diseñar una metodología que permita analizar la situación económica de las poblaciones artesanales, por la incidencia que éstas tienen en la economía nacional. El interés en el tema radica en la escasa información que existe del sector. Para esto, la propuesta considera seis indicadores económicos determinantes en el momento de realizar el análisis, las cuales son: inflación, PIB sectorial, renta disponible, empleo, ventas y, además, se toma en cuenta al indicador financiero nivel de endeudamiento, por el impacto que genera en el desarrollo y crecimiento de las unidades empresariales. Tomando en cuenta que los indicadores son la herramienta más fiable para realizar un análisis económico, 
utilizando una mínima cantidad de datos (Filion, J.; Cisneros L.; Mejía J. 2001. p.322) es apropiado aplicarlo en el análisis de este tipo de poblaciones. Para conceptualizar los indicadores se toman como referencia a varios autores como Mochón (2005) p. 167- p.177; Besley \& Brigham (2000) pp. 53 -118; y Zapata (1996) p. 189, Inec (2010). p.9 (ver Tabla 1).

Tabla 1. Definición de indicadores

\begin{tabular}{|c|c|}
\hline Indicador & Concepto \\
\hline INFLACIÓN & Tasa de variación porcentual que experimentan los precios en un periodo de tiempo. \\
\hline PIB & $\begin{array}{l}\text { Mide el valor monetario total de los bienes y servicios producidos en un país en un } \\
\text { periodo determinado. }\end{array}$ \\
\hline $\begin{array}{l}\text { RENTA } \\
\text { DISPONIBLE }\end{array}$ & $\begin{array}{l}\text { Es la renta personal disponible para el gasto o el ahorro después del pago de } \\
\text { impuestos }\end{array}$ \\
\hline EMPLEO & Se refiere al porcentaje de la población en edad de trabajar. \\
\hline VENTAS & Es el traspaso de propiedad de un bien a un tercero a cambio de dinero \\
\hline $\begin{array}{l}\text { NIVEL } \\
\text { ENDEUDAMIENTO }\end{array}$ & $\begin{array}{l}\text { Mide el porcentaje de los activos financiado por terceros deudas totales / activos } \\
\text { totales }\end{array}$ \\
\hline
\end{tabular}

\section{MATERIALES Y MÉTODOS}

Con el fin de desarrollar una metodología que permita analizar a las poblaciones artesanales con objetividad, se ha utilizado un enfoque mixto, que parte desde el cualitativo y, conforme avanza la investigación, se aborda el cuantitativo. El diseño de la investigación es descriptivo; el instrumento fue la entrevista.

En primera instancia se utilizó la observación pasiva no sistemática con informantes clave, a fin de determinar las necesidades y problemas que, desde su punto de vista, afectan a la población como tal. (ver Figura 1). Se sintetizan los hallazgos en una ficha de observación (ver Tabla 2).

Figura 1. Propuesta metodológica

\section{FASE PRELIMINAR: OBSERVACIÓN}

$\left.\begin{array}{c}\text { Trabajo de campo } \\ \text { observación directa }\end{array} \longrightarrow \begin{array}{c}\text { Levantamiento ficha } \\ \text { observación }\end{array}\right) \longrightarrow \begin{gathered}\text { Análisis e interpretación } \\ \text { de resultados }\end{gathered}$


Tabla 2. Resumen de la ficha de observación

\begin{tabular}{|l|l|l|}
\multicolumn{1}{|c|}{ FASES } & \multicolumn{1}{c|}{ TÉCNICAS } \\
\hline Característica del grupo & $\begin{array}{l}\text { ReSULTADOS } \\
\text { Entrevista; observación. }\end{array}$ & $\begin{array}{l}\text { 1. unidades familiares } \\
\text { 2. informalidad } \\
\text { 3. heterogéneo } \\
\text { 4. nativos de la zona }\end{array}$ \\
\hline $\begin{array}{l}\text { Identificación de los } \\
\text { problemas del grupo }\end{array}$ & $\begin{array}{l}\text { Cualitativa: recolección de datos: } \\
\text { Entrevista; observación. }\end{array}$ & $\begin{array}{l}\text { 1. determinación del costo } \\
\text { 2. competencia } \\
\text { 3. baja demanda de los productos } \\
\text { 4. escases de la materia prima }\end{array}$ \\
\hline $\begin{array}{l}\text { Identificación de las } \\
\text { necesidades }\end{array}$ & $\begin{array}{l}\text { Cualitativa: recolección de datos: } \\
\text { Entrevista; observación. }\end{array}$ & $\begin{array}{l}\text { 1. asociatividad } \\
\text { 2. capacitación } \\
\text { 3. promoción }\end{array}$ \\
\hline $\begin{array}{l}\text { Planificación de la } \\
\text { propuesta }\end{array}$ & cuantitativa: análisis de los datos & $\begin{array}{l}\text { proponer una metodología que permita } \\
\text { analizar la población artesanal en su } \\
\text { conjunto }\end{array}$ \\
\hline
\end{tabular}

Una vez obtenidos los resultados, se procede a su análisis e interpretación. Estos hallazgos son la base para plantear una propuesta metodológica que permita analizar la situación económica de la población de estudio. Se consideran 4 fases que abarcan: 1) determinar la población artesanal; 2) un diagnóstico preliminar; 3) análisis macro y micro económico y 4) un plan de mejora y propuesta.

En todas las fases se distinguen acciones que coadyuvan a cumplir el objetivo de investigación, (ver figura $\mathrm{N}^{\circ} 2$ ). Cada fase debe concluirse antes de proceder a la siguiente. Las fases y sus acciones se describen a continuación.

\section{Propuesta metodológica}

\section{FASE 1: Determinar la población del sector artesanal}

Esta consta de 5 acciones que están orientadas a: planteamiento de objetivos, identificación del número de artesanos, definición de indicadores, diseño del instrumento y aplicación del instrumento.

Acción 1.1: una vez que se ha analizado la ficha de observación, se procede con el planteamiento de objetivos específicos, que son: a). diseñar un modelo metodológico para el análisis económico de la población artesanal, b). Determinar el número de artesanos existentes en la población de estudio, c). analizar la situación económica del sector

Acción 1.2: para cumplir con uno de los objetivos que es determinar el número de artesanos, ya que en la observación se evidenció que, la falta de Asociatividad impide conocer el número exacto, se considera el levantamiento de información de la toda la población de estudio. En este punto se recomienda que a falta de la información documental se realice un censo.

Acción 1.3: se establecen los principales indicadores que ayudarán a analizar la situación económica de la población artesanal. En la metodología se han considerado los siguientes:

- Producto Interno Bruto (PIB) sectorial, mide el valor de mercado de la actividad económica del sector en un año. En este caso se deben tomar las estadísticas del banco Central o de la 
institución responsable de la información económica en un país. Este indicador permite conocer cuánto aporta el sector artesanal. La información ayuda a determinar si la población artesanal tiene un crecimiento o decrecimiento económico en un período determinado.

- Las ventas equivalen al producto por precio, información que debe ser proporcionada por la población que va a ser analizada. Permite medir los ingresos de los artesanos, para esto de utilizará la fórmula: $\mathbf{P V}=\mathbf{C V}+\mathbf{G B V}$, (Zapata. 1996. p. 196). dónde: precio de venta igual al costo de venta más la ganancia bruta en ventas.

- El Empleo bruto, de acuerdo a las recomendaciones de la Organización Internacional del trabajo (OIT) y la Organización para la Cooperación y el Desarrollo Económico (OCED), se refiere a las personas con empleo expresado como porcentaje de la población en edad de trabajar.

TEB $=$ Empleo * 100, (INEC, .2016$)$

PET

En donde PET es la población en edad de trabajar (comprende a las personas de 15 años y más). El indicador de empleo refleja el porcentaje de la población que labora en éste sector.

- La renta personal disponible corresponde a la renta personal menos todos los impuestos y aportaciones sociales, al igual que las ventas los datos se obtienen de la aplicación de la encuesta. Este indicador permite conocer cuánto dinero al año disponen los artesanos para sus gastos y/o ahorro. Para lo cual se utilizará la fórmula descrita por Mochón (2005) p. 180, RPD =C+S. Dónde: consumo es (C) y ahorro (S). Las capacidades de generar ingresos producen bienestar en la población. (INEC. 2016. p.9)

- La inflación es igual al porcentaje de incremento en los costos de producción, este indicador ayuda a conocer la variación porcentual que experimenta el Índice de Precios del Consumidor (IPC) en un periodo, Mochón (2005) p.177. la fórmula es:

\section{inflación 2017 $=\frac{\text { IPC año actual }- \text { IPC año anterior }}{\text { IPC } 2016} * 100$}

de esta manera se obtiene la variación porcentual del IPC en el período dado. El indicador permite analizar la variación en el costo del producto.

- Nivel de endeudamiento, permite conocer el porcentaje de activos de las unidades artesanales que se encuentran comprometidos a terceros. Este porcentaje que es financiado por acreedores debe ser tomado de la información proporcionada por los artesanos. Besley \& Brigham (2000) p.118 indican que la fórmula de cálculo es:

\section{razón de endeudamiento $=\frac{\text { Deudas totales }}{\text { Activos totales }}$}

Acción 1.4: el diseño del instrumento es un punto importante, puesto que se tienen que definir claramente las preguntas, evitando la ambigüedad para obtener las respuestas esperadas, ya que son éstas las que van a proporcionar la información necesaria para calcular los indicadores descritos en la acción 1.3 y el cumplimiento de los objetivos. Aquí se deben abordar temas como: financiamiento, ventas, ingresos, impuestos, volúmenes de producción, costos, etc., las preguntas se adaptarán de acuerdo a las variables e indicadores planteados.

Es importante aclarar que, una vez diseñado el instrumento, éste debe ser validado, con la finalidad de asegurar su confiablidad antes de ser aplicado a la población de estudio. 
Acción 1.5: es la aplicación del instrumento, para alcanzar el objetivo b), se debe aplicar a toda la población. Para esto se debe aprovechar la distribución geopolítica del sector de estudio abarcando todas las zonas rurales y urbanas. Anderson, Sweeney, Williams (2008) definen la recolección de datos en toda una población como censo (p. 16).

Este tipo de recolección de información ayuda a determinar el número real de artesanos y artesanas que están en actividad y de los que abandonaron. Es importante indicar que se necesita un equipo encuestador eficiente, capacitado; debe conocer muy bien el instrumento que va a aplicar con la finalidad de evitar el sesgo de la investigación y la falsedad de la información.

\section{RESULTADOS Y DISCUSIÓN}

\section{FASE 2: Diagnóstico preliminar}

Consta de 5 acciones que van desde la organización de la información, el procesamiento de los datos, la interpretación de los resultados, el diagnóstico preliminar de la situación actual y la selección de la muestra.

Acción 2.1: es importante organizar la información, Kinnear \& Taylor (1998) explican algunos criterios que deben considerarse para clasificar la información antes de ingresarla a la base de datos, pues el investigador tendrá que desarrollar la capacidad de seleccionar las encuestas que son aceptables y desechar las no válidas. De ser el caso, se debe regresar al campo y levantar una nueva encuesta (p.535). Se considera información aceptable a aquella que cumple con los criterios de elegibilidad, integridad, consistencia, exactitud y clarificación de la respuesta, además los instrumentos que están físicamente completos. De esta manera se evitan los datos perdidos que sesgan o empobrecen la investigación.

Acción 2.2: el siguiente paso es el procesamiento de los datos; que se inicia con la codificación de las variables; esto significa que el investigador asigna un símbolo numérico que representa una respuesta específica del instrumento de recolección de datos, (Kinnear \& Taylor, 1998, p.539). una vez codificadas las variables, se procede a la sistematización de los datos, que luego de las correlaciones necesarias entregarán los primeros resultados.

Acción 2.3: los datos por si solos no son de mucha ayuda para la investigación, es información que necesita tener un significado y que resuma la investigación. Por lo tanto, la interpretación consiste en explotar el valor de los datos a su máximo nivel, consiguiendo así las respuestas que se busca satisfacer con la investigación.

Acción 2.4: con la obtención de los primeros resultados, se realiza un diagnóstico preliminar. En esta etapa ya se conoce el número de artesanos y artesanas activos. Además, los datos básicos que ayudan a medir los indicadores para su posterior análisis.

Acción 2.5: una vez determinado el número de artesanos, y con la finalidad de triangular los resultados, se procede a calcular una muestra que representará a la población de estudio. En este punto es importante tomar en cuenta que, si la población artesanal se dedica a una sola actividad se tomará una sola muestra. Pero si existen varias actividades artesanales, se debe tomar una muestra estratificada, logrando representar equitativamente a cada grupo artesanal.

\section{FASE 3: Análisis Macro y Microeconómico}

La componen 7 acciones: segmentación de los participantes para grupos focales, diseño del instrumento aplicación del mismo a los grupos focales, análisis micro, análisis macro, procesamiento de la información e interpretación de los resultados y diagnóstico final con el análisis económico.

Acción 3.1: existen poblaciones artesanales heterogéneas en las cuales se distinguen varios tipos de artesanías, en este caso se debe segmentar la población por rama artesanal. Para esto se recomienda el muestreo estratificado que permite una selección proporcional de cada grupo identificado. Después se 
forman los grupos focales para obtener la información de primera mano con la aplicación de un cuestionario de entrevista, Estarán formados por, no más, de 7 personas y de acuerdo al número de artesanos existentes en el sector.

Acción 3.2: en esta etapa se diseña el instrumento para los grupos focales. Se recomienda un cuestionario de entrevista, aquí se abarcarán con detalle todos los aspectos que lleven ala análisis del micro entorno.

Acción 3.3: corresponde a la aplicación de la entrevista, se deben abarcar los temas de volúmenes de producción, precios de venta, instituciones de financiamientos, etc. Es decir, lograr obtener respuestas que nos permitan medir los indicadores económicos planteados en la acción 1.3. Además, se debe aprovechar este diálogo para conocer desde el punto de vista del artesano las debilidades y fortalezas con las que cuentan las unidades productivas. Esta información será de ayuda para formular las estrategias al final de la propuesta.

Acción 3.4: en esta etapa se procede al análisis micro económico. Es de vital importancia identificar los recursos materiales, financieros y humanos, producto, procesos, ventas, ingresos, etc.

Acción 3.5: el análisis macro económico corresponde a revisar estadísticas sobre el PIB, inflación y empleo, para poder conocer la participación del sector artesanal a nivel nacional

Acción 3.6: al igual que en la acción 2.2, es necesario procesar la información obtenida en las sesiones de los focus group, para su posterior interpretación y análisis. Una vez más, el investigador debe seleccionar la información que se procesará, identificando la validez y asegurando la confiabilidad en los datos.

Para esto Hamui \& varela (2012) indican que se debe apoyar en una bitácora que registre todas las actividades realizadas con los grupos, además recomiendan el uso de videocámaras, fotografías, entre otros.

Acción 3.7: se procede a realizar el diagnóstico final de la situación actual de los artesanos del sector, que es el resultado del diagnóstico preliminar más la información proporcionada por los artesanos en los grupos focales, más las estadísticas obtenidas de las instituciones responsables de la información económica del país.

Toda la información recolectada en las acciones anteriores permite realizar el análisis económico del sector. Los datos recolectados son la base para el cálculo y posterior medición de los indicadores económicos.

En el escenario de una estabilidad de la inflación y las tasas de interés, donde las mediciones reflejen un incremento del PIB sectorial y el aumento en la renta personal disponible de los artesanos, se evidenciará un crecimiento económico del sector artesanal.

Si las condiciones son opuestas, se evidenciará el efecto contrario; porque tanto, la inflación como las tasas de interés afectan directamente a los costos de producción.

\section{FASE 4: Plan de mejora y propuesta}

Acción 4.1: el investigador tomará como base los resultados de las fases anteriores y con los datos reales brindados por los artesanos, pasará a la etapa de formular estrategias que permitan dinamizar economía del sector. 
FASE 1: Determinar la población artesanal del sector de estudio

Acción 1.1

Planteamiento de Objetivos

Acción 1.5

Aplicación del instrumento

FASE 2: Diagnóstico Preliminar

Organizar la
información

FASE 3: Análisis: Macro y Microeconómico

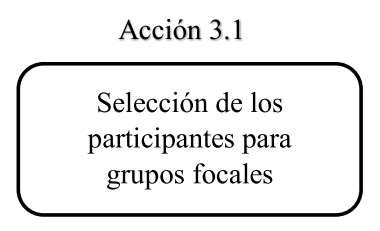

Acción 3.5

Análisis Macro

PIB, Inflación, empleo
Acción 1.2

Investigar el número artesanos existentes
Acción 1.3

Definición de las variables e indicadores
Acción 1.4

Diseño del instrumento: (ENCUESTA)
Acción 2.4

Diagnóstico preliminar de la situación actual 


\section{CONCLUSIONES}

La propuesta metodológica constituye una herramienta fundamental para analizar económicamente poblaciones artesanales, previo a la formulación de estrategias que permitan dinamizar la economía en un sector.

Los indicadores económicos utilizados en la propuesta, son los principales indicadores que reflejan el crecimiento o desarrollo de un sector en un periodo determinado.

La propuesta metodológica contiene las acciones básicas para alcanzar el objetivo de la investigación y han sido dispuestas de acuerdo a la observación y necesidades determinadas por el investigador.

Con la finalidad de evitar sesgos en la metodología o en los datos, el modelo metodológico, está diseñado para triangular los resultados, a fin de obtener datos viables y confiables.

La presente propuesta brinda la oportunidad a nuevos investigadores de aplicarla en las diversas poblaciones artesanales contribuyendo a la mejora de la misma.

\section{REFERENCIAS BIBLIOGRÁFICAS}

Anderson, D., Sweeney, D., Williams, T. (2008). Estadística para administración y Economía. (10ma ed.). México: Cengage Learning, p.16.

Besley, S., Brigham, E. (2000). Fundamentos de administración Financiera. (5ta ed.) México D.F.: Mc Graw Hill. pp. 53- 118.

Castro, E. (2010). Las estrategias competitivas y su importancia en la buena gestión de las empresas. Ciencias Económicas, 28(1), 247 - 276

Culkin, N. \& Smith, D. (2000). An Emotional Business. A guide to understanding the motivations of small business decicion takers. Qualitativa market research.

Filión, J.; Cisneros, L.; Mejía, J. (2011). Administración de Pymes: Emprender, dirigir y desarrollar empresas. México: Pearson

Hamui, A. \& Varela, M. (2013). Metodología de Investigación en Educación Médica: La técnica de grupos focales. Inv. Ed. Med. Elsevier. 2(1), 55-60.

Hernández, R., Fernández, C., Baptista, P. (2010). Metodología de la Investigación. 5ta edición. México D.F.: Mc. Graw Hill p.203.

Instituto Nacional de Estadísticas y Censos (2016). Metodología para la medición del empleo en el Ecuador. Quito. p. 2-22

Kinnear T., Taylor J, (1998). Investigación de Mercados. 5ta edición. Colombia: Mc. Graw Hill. pp.301 - 539.

Landázuri Espinoza, S.N.; Montenegro Cazares, N.J. (2018). El enfoque estratégico de Michael Porter aplicado a las Mipymes: Caso Ibarra - Ecuador. Hallazgos 21, suplemento especial, 3, 1-8.

Leff, E. (2003). Ecología y capital. Racionalidad ambiental, democracia participativa y desarrollo sustentable. Buenos Aires. Siglo XXI. p.140 a 258

Mochón, F. (2005). Principios de Economía. (3ra Ed.). Madrid. Mc Graw Hill, p 167- p180.

Munch, L., Ängeles, E. (1997). Metodología e la Investigación. México: Editorial Trillas, p 19.

Oliveras, M.L; Albanese, V. (2012) Etnomatemáticas en Artesanías de Trenzado: un modelo metodológico para investigación Boletim de Educação Matemática, Universidad de Estadual Paulista Júlio de Mesquita Filho Rio Claro. Brasil, 26(44), 1315-1344

Pacheco, J.; Gómez, G.; Barrero, G. (2009). El desafío de las comunidades artesanales rurales: una propuesta ecotecnológica para una artesanía sostenible. Acta Agronómica. 58(3), 206 - 220

UNESCO (2010). Informe Mundial: Invertir en la diversidad cultural y el diálogo intercultural. p. 180.

Zapata, P. (1999). Contabilidad General. (2da ed.). Colombia. Mc Graw Hill. p.189. 